\title{
Bell's Non-Locality Theorem Can Be Understood in Terms of Classical Thermodynamics
}

\author{
Tomofumi Miyashita \\ Miyashita Clinic, Mitsuya-kita, Yodogawa-ku, Osaka, Japan \\ Email: tom_miya@plala.or.jp
}

How to cite this paper: Miyashita, T. (2017) Bell's Non-Locality Theorem Can Be Understood in Terms of Classical Thermodynamics. Journal of Modern Physics, 8 , 87-98.

http://dx.doi.org/10.4236/jmp.2017.81008

Received: December 30, 2016

Accepted: January 17, 2017

Published: January 20, 2017

Copyright $\odot 2017$ by author and Scientific Research Publishing Inc. This work is licensed under the Creative Commons Attribution International License (CC BY 4.0).

http://creativecommons.org/licenses/by/4.0/ (c) (i) Open Access

\begin{abstract}
Bell's non-locality theorem can be understood in terms of classical thermodynamics, which is already considered to be a complete field. However, inconsistencies in classical thermodynamics have been discovered in the area of solid-oxide fuel cells (SOFCs). The use of samarium-doped ceria electrolytes in SOFCs lowers the opencircuit voltage (OCV) to less than the Nernst voltage. This low OCV has been explained by Wagner's equation, which is based on chemical equilibrium theory. However, Wagner's equation is insufficient to explain the low OCV, which should be explained by fluctuation and dissipation theorems. Considering the separation of the Boltzmann distribution and Maxwell's demon, only carrier species with sufficient energy to overcome the activation energy can contribute to current conduction, as determined by incorporating different constants into the definitions of the chemical and electrical potentials. Then, an energy loss equal to the activation energy will occur because of the interactions between ions and electrons. This energy loss means that an additional thermodynamic law based on an advanced model of Maxwell's demon is needed. In this report, the zero-point energy can be explained by this additional thermodynamic law, as can Bell's non-locality theorem.
\end{abstract}

\section{Keywords}

Wagner's Equation, Fluctuation and Dissipation Theory, Boltzmann Distribution, Maxwell's Demon, Additional Thermodynamic Law

\section{Introduction}

Solid-oxide fuel cells (SOFCs) directly convert the chemical energy of fuel gases, such as hydrogen and methane, into electrical energy. SOFCs use a solid-oxide film as the electrolyte, and oxygen ions serve as the main charge carriers. Typically, yttria-stabilized zirconia (YSZ) is used as the electrolyte material in these cells. The operating temperature of these cells ( $873-1273 \mathrm{~K}$ ) should be reduced to extend their life spans. Therefore, the use of mixed ionic and electronic conducting (MIEC) electrolyte materials, such as 
samaria-doped ceria (SDC) electrolytes, at lower temperatures is preferred. The opencircuit voltage (OCV) of an SDC cell is approximately $0.8 \mathrm{~V}$, which is lower than the Nernst voltage $\left(V_{\mathrm{th}}\right)$ of $1.15 \mathrm{~V}$ at $1073 \mathrm{~K}$. This low OCV is attributed to the low value of the ionic transference number $\left(t_{\text {ion }}\right)$. The low OCV has been calculated using Wagner's equation, which is based on chemical equilibrium theory [1] [2]. However, there are serious problems in Wagner's equation [3]-[8]. We discuss these problems in Section 2.

Over the past two decades, the understanding of nonequilibrium thermodynamics has been enhanced by fluctuation and dissipation theorems such as the Jarzynski and Crooks relations [9] [10]. Attempts have been made to explain Maxwell's demon based on fluctuation and dissipation theorems [11]. We discovered the following empirical equation using SDC electrolytes [12]:

$$
\mathrm{OCV}=V_{\mathrm{th}}-\frac{E_{a}}{2 \mathrm{e}}
$$

where $E_{a}$ is the ionic activation energy, which is $0.7 \mathrm{eV}$ for SDC electrolytes. Therefore, the OCV in Equation (1) is $0.80 \mathrm{~V}(=1.15 \mathrm{~V}-0.7 \mathrm{eV} / 2 \mathrm{e})$. Equation (1) can be explained using an advanced model of Maxwell's demon. On the basis of this model, we proposed an additional thermodynamic law. We previously discussed the relationship between quantum physics and this additional thermodynamic law [8]. In Section 3, we discuss a prototypical and an advanced model of Maxwell's demon in relation to hopping conduction.

Then, the inevitable energy dissipation equal to $1 / 2 \hbar \omega$ from the particles to the environment will occur as the zero-point energy. Consequently, the space uses not the prototypical model of Maxwell's demon using YSZ electrolytes but rather the advanced model of Maxwell's demon using SDC electrolytes. In Section 4, we discuss the relationship between the zero-point energy and this additional thermodynamic law.

Bell's theorem [13] is a "no-go theorem" that draws a distinction between quantum mechanics and classical mechanics. Bell's theorem states that "No physical theory of local hidden variables can reproduce the predictions of quantum mechanics." However, Bell did not know that the information of local hidden variables could be erased during measurement due to the inevitable energy dissipation. In Section 5, based on the advanced model of Maxwell's demon, we show that Bell's non-locality theorem can be explained in terms of classical thermodynamics. Furthermore, a determination experiment to disprove quantum teleportation is proposed. Bell's non-locality theorem can be local with this additional thermodynamic law.

\section{Problems in Chemical Equilibrium Theory}

The low OCV has been explained by Wagner's equation, which is based on chemical equilibrium theory. The flux of oxygen ions under open-circuit conditions, as described by Wagner's equation [1] [2], is

$$
J_{O_{2}}=-\frac{R T}{16 F^{2} L} \int_{\ln p O_{2}^{\text {nande }}}^{\ln p O_{\text {cathode }}^{\text {al }}} \frac{\sigma_{\mathrm{el}} \sigma_{\text {ion }}}{\sigma_{\text {el }}+\sigma_{\text {ion }}} \mathrm{d} \ln p O_{2}
$$

where $\mathrm{J}_{\mathrm{O}_{2}}$ and $\mathrm{pO}_{2}$ are the $\mathrm{O}_{2}$ flux and the $\mathrm{O}_{2}$ partial pressure, respectively; $p \mathrm{O}_{2}^{\text {cathode }}$ and $\mathrm{pO}_{2}^{\text {anode }}$ are the $\mathrm{O}_{2}$ partial pressures at the cathode and the anode, re- 
spectively; $R, T$, and $F$ are the gas constant, the absolute temperature, and Faraday's constant, respectively; $L$ is the thickness of the membrane or film; and $\sigma_{\text {el }}$ and $\sigma_{\text {ion }}$ are the conductivities of the electrons and oxygen vacancies, respectively.

From Equation (2), Equations (3) and (4) can be deduced [14].

$$
\mathrm{OCV}=V_{\mathrm{th}}-R_{i} I_{i}
$$

where $R_{i}$ and $I_{i}$ are the ionic resistances of the electrolyte and the ionic current, respectively.

$$
\mathrm{OCV}=\frac{R T}{4 F} \int_{\ln p O_{2}^{\text {anode }}}^{\ln p \mathrm{O}_{\text {ion }}^{\text {cathode }}} \mathrm{d} \ln p O_{2} .
$$

Equation (4) is often used when $\sigma_{\text {ion }}$ is constant under various $O_{2}$ partial pressures [14], and $I_{i}$ is not apparently expressed. When an SOFC is operated under OCV conditions, the electronic leakage current $\left(I_{e}\right)$ is defined using the external current $\left(I_{\text {ext }}\right)$ :

$$
I_{\text {ext }}=I_{i}+I_{e}=0 .
$$

In general, $I_{i}$ and $I_{e}$ are not zero. Parameter $t_{\text {ion }}$ is expressed as

$$
t_{\text {ion }}=\frac{\sigma_{\text {ion }}}{\sigma_{\text {el }}+\sigma_{\text {ion }}} .
$$

However, $\sigma_{\text {el }}$ is a function of the $O_{2}$ partial pressure [15]:

$$
\sigma_{\text {el }}=\sigma_{\text {ion }}\left(\frac{p O_{2}}{p O_{2}^{*}}\right)^{-\frac{1}{4}}
$$

where $p O_{2}^{*}$ corresponds to the oxygen partial pressure at which $t_{\mathrm{ion}}=1 / 2$. However, Wagner's equation encounters the following problems.

Problem 1: The calculated OCV will always be higher than the experimental result. Polarization voltage losses are frequently used to compensate, but the lack of change of the OCV during electrode degradation is impossible to explain [3] [4] [5].

Problem 2: The equilibration process of thick SDC electrolytes in response to a change in the anode gas cannot be explained because the response from the experimental results is too fast to explain the delay in the electron diffusion current from the cathode to the anode [6].

To solve the aforementioned problems, there should be a fast response currentindependent constant anode voltage loss $(0.35 \mathrm{~V}=1.15 \mathrm{~V}-0.80 \mathrm{~V})$ [5] [6]. Then, $R_{i} I_{i}$ becomes very small because of the anode shielding effect [5] [6] [16].

\section{Prototypical and Advanced Model of Maxwell's Demon in Relation to Hopping Conduction}

\subsection{Prototypical Model of Maxwell's Demon in Relation to Hopping Conduction}

YSZ is a ceramic in which the crystal structure of zirconium dioxide is made stable by the addition of yttrium oxide. When yttria is added to pure zirconia, $\mathrm{Y}^{3+}$ ions replace $\mathrm{Zr}^{4+}$ on the cationic sublattice. Oxygen vacancies are generated due to the charge neutrality. A graphical explanation is provided in Figure 1. Ionic conduction involves 


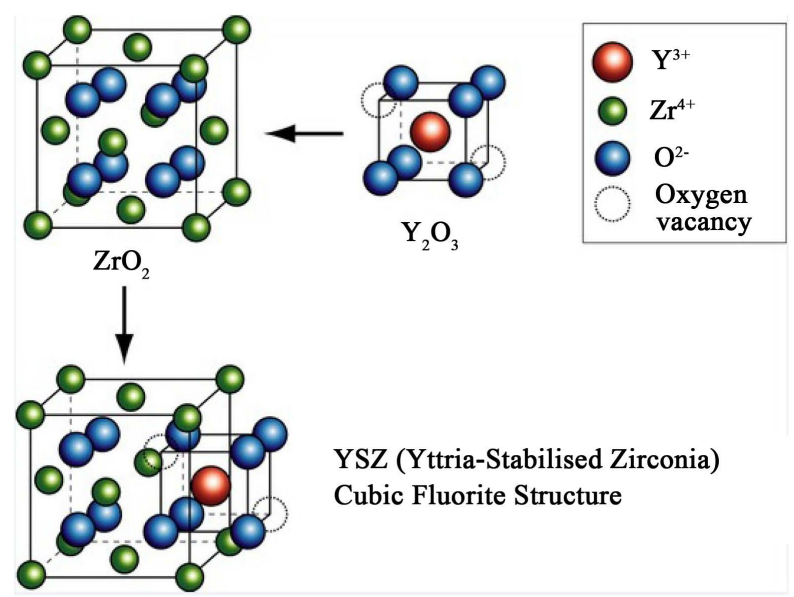

Figure 1. Graphical explanations for generating oxygen vacancies. Oxygen vacancies are generated because of charge neutrality. Quoted from Yttria-stabilized zirconia (Wikipedia).

https://en.wikipedia.org/wiki/Yttria-stabilized_zirconia

hopping from an occupied site (8c site) to a vacant lattice site (8c). Oxygen ions then pass through the first gate ( $32 \mathrm{f}$ site) and the second gate (another $32 \mathrm{f}$ site) [17]. A schematic of the path for ions is shown in Figure 2. The situation is very similar to that of a prototypical model of Maxwell's demon. Landauer first proposed the principle of this model in 1961. Landauer's principle states that, if an observer loses information about a physical system, the observer loses the ability to extract work from that system [18].

\subsection{Advanced Model of Maxwell's Demon in Relation to Hopping Conduction}

YSZ electrolytes are purely ionic conductors; however, SDC electrolytes are MIEC materials. Interactions should occur between hopping ions and electrons. A schematic of the path for ions in SDC electrolytes is shown in Figure 3. Gabor and Brillouin independently proposed the energy loss during interactions [19] [20]. Hence, the situation is different from the use of YSZ electrolytes, which can be explained by Landauer's principle [18].

In previous work, we explained the advanced model of Maxwell's demon electrochemically [6] [8]. The Boltzmann distribution of oxygen ions in the electrolyte at 1073 $\mathrm{K}$ is displayed in Figure 4. The ions with energies exceeding the ionic activation energy become carriers (hopping ions). Figure 5 presents an incorrect carrier distribution. The Boltzmann distribution cannot be separated using passive filters, and an accurate distribution is provided in Figure 6. The loss of Gibbs energy is illustrated in Figure 5, and the real Gibbs energy ( $\left.G_{\text {real }}\right)$ should be determined using Equation (8) [6]:

$$
\Delta G_{\text {real }}=\Delta G-N E_{a}
$$

Then, voltage losses occur during every hopping process in the MIEC area. Therefore, the voltage loss resulting from only four hopping processes $(1.4 \mathrm{~V}=0.35 \times 4 \mathrm{~V})$ should be higher than the Nernst voltage $(1.15 \mathrm{~V})$. However, the voltage loss is attributed to only one hopping process. This voltage loss can be explained by the Jarzynski equality [6]. In thermodynamics, the free energy difference $\left(\Delta F=F_{B}-F_{A}\right)$ between two states $A$ and $B$ is connected to the work $W$ through the inequality 


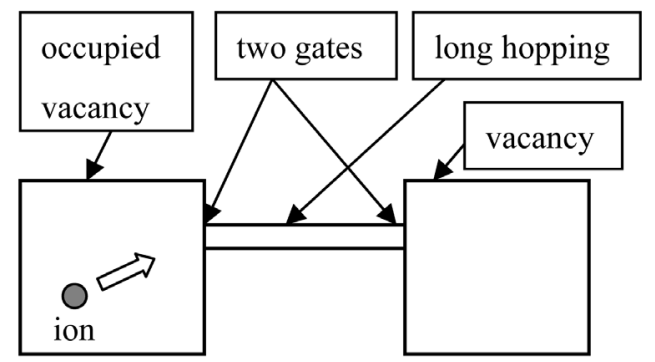

Figure 2. Schematic of the path for ions in YSZ electrolytes. The situation is very similar to that of a prototypical model of Maxwell's demon explained by Landauer's principle.

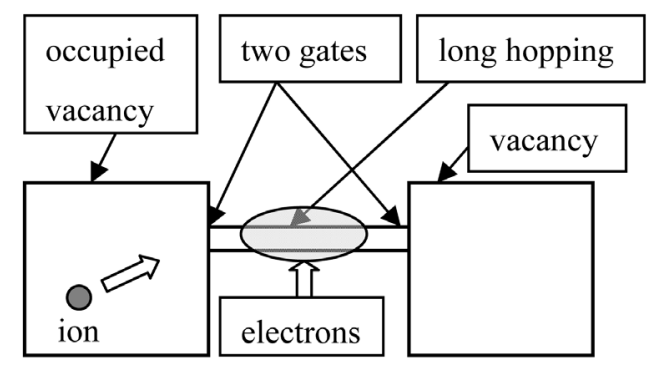

Figure 3. Schematic of the path for ions in SDC electrolytes. Interactions should exist between the hopping ions and electrons, as explained by Gabor and Brillouin.

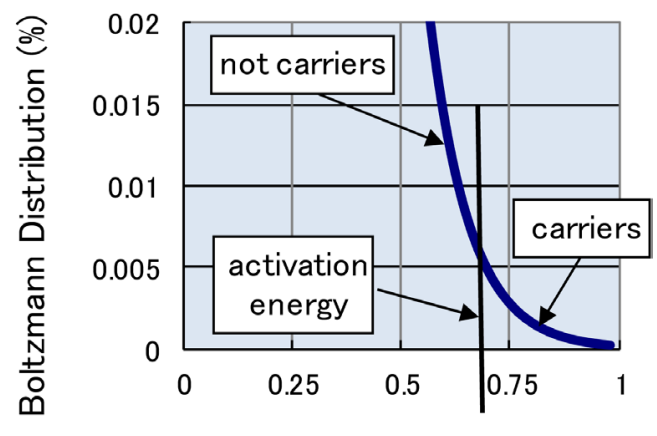

Ion's Electrochemical Potential

per mol, when $\varphi$ is zero $(e \mathrm{~V})$

Figure 4. Boltzmann distribution at $1073 \mathrm{~K}$. Ions with energies exceeding the ionic activation energy are converted into charge carriers (i.e., hopping ions).

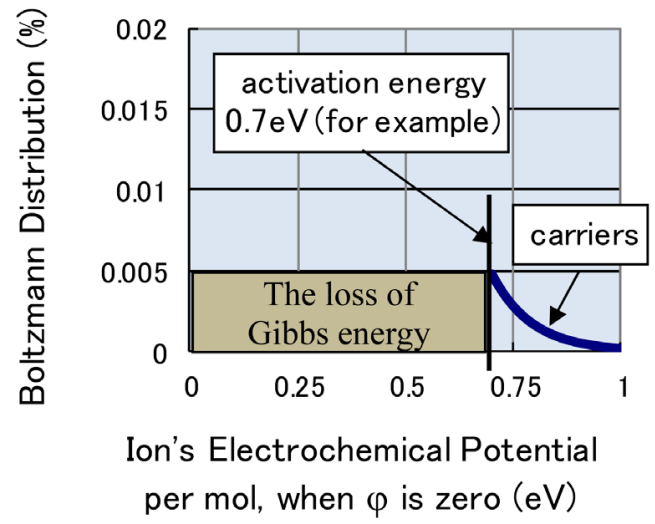

Figure 5. Forbidden distribution of hopping ions. This distribution is forbidden according to "Maxwell's demon." 


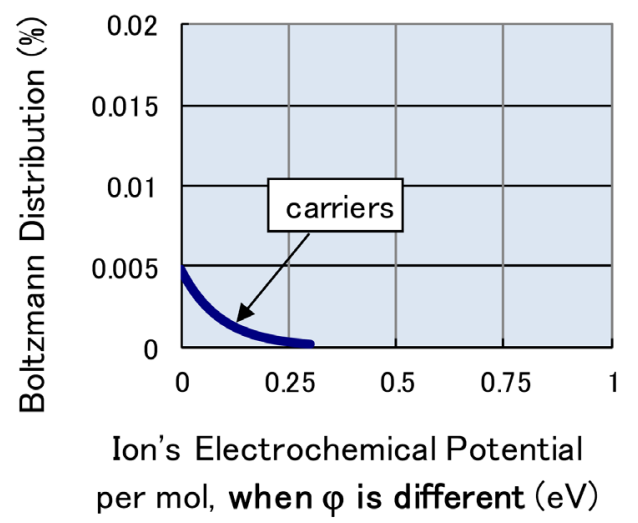

Figure 6. Correct distribution of hopping ions. The shape of the distribution in this figure should be the same as the shape of the distribution in Figure 4.

$$
\Delta F \leq W
$$

The Jarzynski equality is [7]

$$
\mathrm{e}^{-\Delta F / K T}=\mathrm{e}^{\overline{-W / K T}}
$$

where the over-line indicates an average over all possible realizations of an external process that takes the system from the equilibrium state $A$ to a new nonequilibrium state under the same external conditions as that of the equilibrium state $B$.

Ions in equilibrium state $A$ lose energy $(=0.7 \mathrm{eV})$ during their hopping in the MIEC electrolyte, and the heating of the colder ions can occur immediately in the vacancies. However, after the last hop, the ions cannot be heated and therefore exit the electrolyte in equilibrium state $B$. $A$ schematic of this explanation of the energy loss is shown in Figure 7. Consequently, Equation (1) is compatible with the second law of thermodynamics.

\subsection{Additional Thermodynamic Law Based on the Advanced Model of Maxwell's Demon}

The electrochemical potential can be separated into the chemical potential and the electrostatic potential,

$$
\eta_{i}=\mu_{i}+z_{i} F \varphi
$$

where $Z_{i}, \eta_{i}, \mu_{i}$ and $\varphi$ are the valence of species $i$, the electrochemical potential, the chemical potential and the electrostatic potential. In Equation (11), the following transformation should be considered during ion hopping:

$$
\begin{gathered}
\mu_{i_{-} \text {hopping }}=\mu_{i_{-} \text {vacancies }}+N E_{a} \\
Z_{i} F \varphi_{\text {hopping }}=Z_{i} F \varphi_{\text {vacancies }}-N E_{a}
\end{gathered}
$$

where $N, \mu_{i_{-} \text {hopping }}, \mu_{i_{-} \text {vacancies }}, \varphi_{i_{-} \text {hopping }}$, and $\varphi_{i_{-} \text {vacancies }}$ are Avogadro's number, the chemical potential of hopping ions, the chemical potential of ions in vacancies, the electrical potential of hopping ions, and the electrical potential of ions in vacancies, respectively. Thus, when $E_{a}$ is $0.7 \mathrm{eV}$,

$$
\varphi_{\text {hopping }}=\varphi_{\text {vacancies }}+\frac{E_{a}}{2 \mathrm{e}}=\varphi_{\text {vacancies }}+0.35 \mathrm{~V}
$$




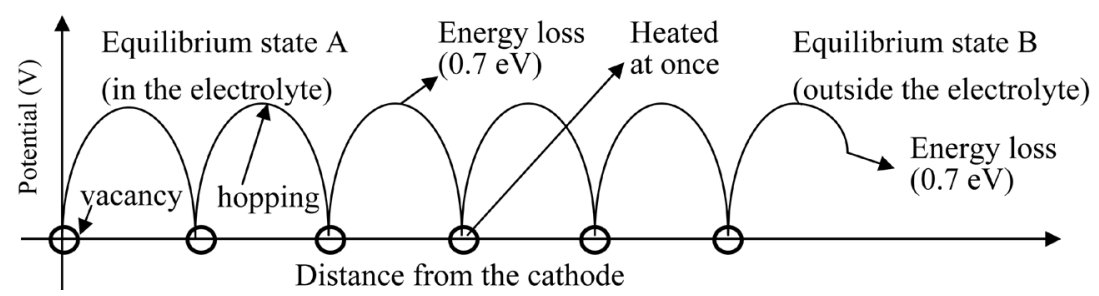

Figure 7. Schematic of the explanation for the energy loss. Equation (1) can be compatible with the Jarzynski equality.

The electrical potential $(0.35 \mathrm{~V}=0.7 \mathrm{eV} / 2 \mathrm{e})$ in Equation (14) is neutralized by free electrons. Thus, our proposed additional thermodynamic law based on the advanced model of Maxwell's demon is stated as follows:

"Considering the separation of the Boltzmann distribution and Maxwell's demon, only carrier species with sufficient energy to overcome the activation energy can contribute to current conduction, as determined by incorporating different constants into the definitions of the chemical and electrical potentials. Then, an energy loss equal to the activation energy will occur because of the interactions between ions and electrons."

Using $S$ (the action function), we can state the Nernst-Planck equation as

$$
S=\int\left(\mu_{i}-Z F \varphi\right) \mathrm{d} t
$$

From the principle of least action,

$$
\delta S=0 .
$$

Considering the separation of the Boltzmann distribution and Maxwell's demon, the generalized expression obtained from Equations (12) and Equation (13) is

$$
\frac{\mathrm{d} S}{\mathrm{~d} t}=\mu_{i}-Z F \varphi=2 N E_{a} .
$$

Equation (17) holds for one hop but not zero when $E_{a}$ is not zero. In SDC electrolytes, the energy loss during hopping due to inevitable dissipation $\left(E_{\text {loss }}\right)$ is

$$
E_{\text {loss }}=N E_{a}=\frac{1}{2} \frac{\mathrm{d} S}{\mathrm{~d} t} \text {. }
$$

\subsection{Determination Experiment for an Advanced Model of Maxwell's Demon}

Oxygen ions transport through a path during hopping in the lattice structure. The electric potential in the path during hopping differs from the electric potential in the vacancies. In YSZ electrolytes, which are purely ionic conductors, ions do not lose energy. However, in SDC electrolytes, which are MIEC materials, the ions lose a large amount of energy. However, measuring the electric potential during hopping is difficult. We thus propose a determination experiment. A negatively charged metal ball (N-ball) and positively charged heavy metal balls (P-balls) should all be coated with plastic. Using springs, the three P-balls can be connected with each other, and then the N-ball can go through the center of them at high speed under vacuum. The speed of the N-ball may be unchanged after the collision. However, when an ionized gas spray is used during the collision, the speed of the $\mathrm{N}$-ball will become small. The energy loss of the N-ball 
can be controlled by changing the spring constant. A schematic of the explanation of this determination experiment is shown in Figure 8.

\section{Explanation of the Zero-Point Energy in Terms of Classical Thermodynamics}

Equation (17) and Equation (18) form the generalized expression of the additional thermodynamic law. In quantum physics, instead of $\mu_{i}, Z F \varphi$ and $2 N E_{a}, K . E$. (kinetic energy), P.E. (potential energy) and $\hbar \omega$ are used; the energy for one particle is [21]

$$
\frac{\mathrm{d} S}{\mathrm{~d} t}=K \cdot E \cdot-P \cdot E \cdot=-\hbar \omega
$$

where $\hbar$ is Planck's constant and $\omega$ is the frequency. The zero-point energy $\left(E_{0}\right)$ for one dimension is [21]

$$
E_{0}=-\frac{1}{2} \hbar \omega=\frac{1}{2} \frac{\mathrm{d} S}{\mathrm{~d} t}
$$

Equation (20) indicates that, when the separation of the Boltzmann distribution by $1 / 2 \hbar \omega$ is needed, Maxwell's demon should be considered. Here, $1 / 2 \hbar \omega$ should be a boundary belonging to space and not to the particles to separate the observed particles from the unobserved particles. The situation is analogous to that in which $E a$ is a boundary for the lattice structure rather than for ions [8]. Parameter $\hbar$ is assigned to the fifth spatial dimension. Then, the inevitable energy dissipation equal to $1 / 2 \hbar \omega$ from the particles to the environment will occur as the zero-point energy. Consequently, the space uses not the prototypical model of Maxwell's demon using YSZ electrolytes but rather the advanced model of Maxwell's demon using SDC electrolytes.

\section{Bell's Non-Locality Theorem Can Be Understood in Terms of Classical Thermodynamics}

\subsection{Bell's Non-Locality Theorem}

Bell's theorem is a "no-go theorem" that draws a distinction between quantum mechanics and classical mechanics [13]. Bell's theorem states, "No physical theory of local hidden variables can reproduce the predictions of quantum mechanics."

The inequality that Bell derived can then be written as

$$
C_{h}(a, c)-C_{h}(b, a)-C_{h}(b, c) \leq 1
$$

where $C_{h}(a, b)$ denotes the correlation as predicted by any hidden variable theory and $a, b$ and $c$ refer to three arbitrary settings of the two analyzers. This theorem is

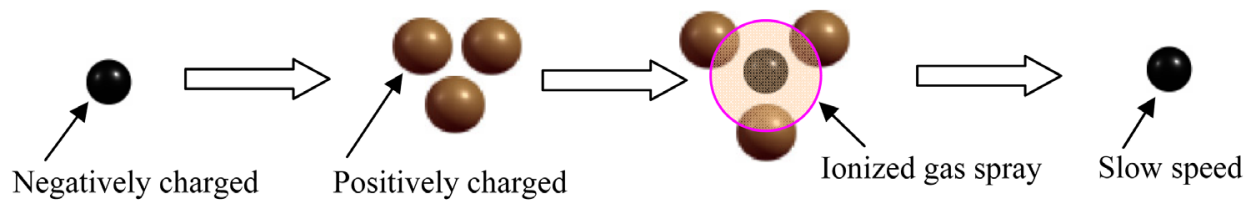

Figure 8. Schematic of the explanation of the determination experiment for an additional thermodynamic law. When an ionized gas spray is used during the collision, the speed of the N-ball will become small. The energy loss of the negatively charged metal ball can be controlled by changing the spring constant. 
proven by considering a system of two entangled qubits. The examples concern systems of particles that are entangled in spin. Quantum mechanics allows predictions of correlations that would be observed if these two particles have their spin measured in different directions.

However, Bell was unaware of the inevitable energy dissipation during the measurement. According to Brillouin [20], information means negative entropy (negentropy), which should be erased during the observation. Hence, the information of local hidden variables can be erased during the inevitable energy dissipation.

\subsection{Confounding Factor of the Inevitable Energy Dissipation}

In statistics, a confounding factor is an extraneous factor in a statistical model that correlates with both the dependent variable and the independent variable in a way that explains away some of the correlation between these two variables.

When there should be inevitable energy dissipation from particles to the environment during the measurement, combined with the relative theory, the total angular momentum of two entangled qubits cannot be conserved after the measurement. We propose the following confounding factor based on locality.

Confounding factor: Spin is similar to a vector quantity that has a direction as the local hidden variable. However, the information of the direction can be erased because of the inevitable energy dissipation during the measurement. This information refers to spin in the positive or negative direction of the chosen axis after the measurement.

\subsection{Calculation of Correlation Coefficient Considering the Confounding Factor Based on Locality}

We consider a photon. The frequency of photons is much higher than that of radio waves. Within radio waves, AM broadcasting is the process of radio broadcasting using amplitude modulation and FM broadcasting uses frequency modulation. In principle, we can manipulate photons using amplitude modulation and frequency modulation. When the angle between a vector quantity and the chosen axis is $\theta_{0}$, amplitude modulation is achieved by changing $\cos \theta_{0}$ and frequency modulation is achieved by changing $\sin \theta_{0}$. Then, the rest of the information $\left(I_{0}\right)$, deleting the amplitude modulation and frequency modulation, can be defined using the following equation:

$$
I_{0}=1-\left(\cos \theta_{0}+i \sin \theta_{0}\right)=1-\mathrm{e}^{i \theta_{0}}=\int_{\theta_{0}}^{0} \frac{\mathrm{e}^{\mathrm{i} \theta}}{i} \mathrm{~d} \theta
$$

Then, the rest of the information $I_{1}$ between $\theta_{0}$ and $\theta_{1}$ is

$$
I_{1}=\int_{\theta_{0}}^{0} \frac{\mathrm{e}^{i \theta}}{i} \mathrm{~d} \theta-\int_{\theta_{1}}^{0} \frac{\mathrm{e}^{i \theta}}{i} \mathrm{~d} \theta=\int_{\theta_{0}}^{\theta_{1}} \frac{\mathrm{e}^{i \theta}}{i} \mathrm{~d} \theta
$$

Thus, $I_{1}$ is determined only by $\theta_{0}$ and $\theta_{1}$. Equation (23) contains a rotational symmetry element; thus, the correlation coefficient should be determined by $\theta_{1}-\theta_{0}$, which means that we must consider only the function of the correlation coefficient in Equation (22). This result is obtained by considering a confounding factor due to the inevitable energy dissipation. When a perfect polarizer is placed in a polarized beam of light, Malus' law in classical physics is 


$$
C_{h}\left(0, \theta_{0}\right)=\cos ^{2} \theta_{0}
$$

In general, because of rotational symmetry, Equation (24) should be rewritten as

$$
C_{h}\left(\theta_{1}, \theta_{0}\right)=\cos ^{2}\left(\theta_{1}-\theta_{0}\right)
$$

In the experiment performed by Aspect [22], the definition of $\theta$ is $\pi / 2-\theta$. Then,

$$
C_{h}\left(\theta_{1}, \theta_{0}\right)=\sin ^{2}\left(\theta_{1}-\theta_{0}\right)
$$

Consequently, Bell's non-locality theorem can be understood in terms of classical thermodynamics.

\subsection{Determination Experiment for Local Hidden Variables Considering a Confounding Factor Based on Locality}

We proposed a determination experiment for local hidden variables, which is combined with the experiment of Aspect and a double-slit experiment. The source $S$ produces pairs of photons, which are sent in opposite directions. After the detection of the left photons, the right photon receives the information of its position by quantum teleportation. Then, an interference pattern cannot be created on the screen behind the double slit. A schematic of the determination experiment for the local hidden variables is shown in Figure 9. When an interference pattern is observed, quantum teleportation (non-locality) will be denied.

\section{Summary}

Inconsistencies in classical thermodynamics have been discovered in the area of SOFCs. The use of SDC electrolytes in SOFCs lowers the OCV to less than the Nernst voltage. This low OCV has been explained by Wagner's equation, which is based on chemical equilibrium theory. However, Wagner's equation is insufficient to explain the low OCV. By considering the separation of the Boltzmann distribution and Maxwell's demon, we proposed an additional thermodynamic law based on an advanced model of Maxwell's demon. The zero-point energy can be explained by this additional thermodynamic law. Then, inevitable energy dissipation equal to $1 / 2 \hbar \omega$ from the particles to the environment will occur as the zero-point energy. Consequently, the space uses not the prototypical model of Maxwell's demon, but rather the advanced model of

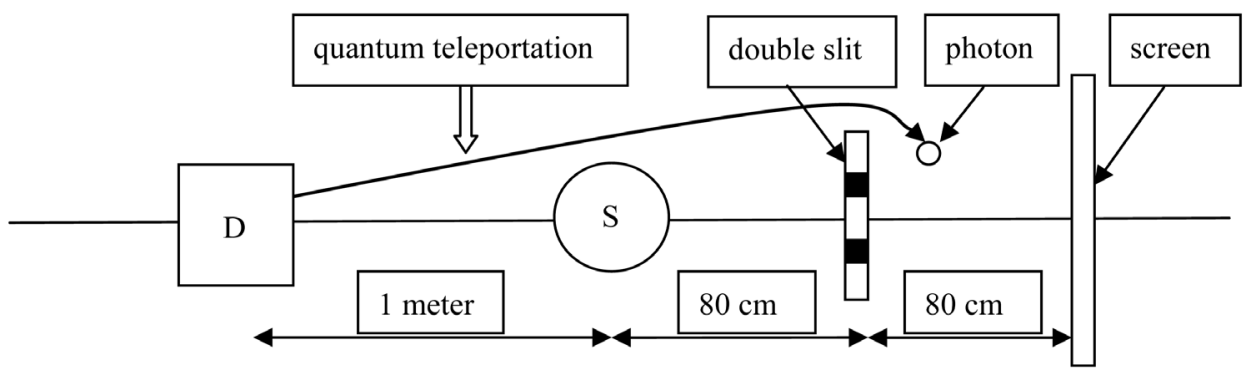

Figure 9. Schematic of the determination experiment for local hidden variables. The source S produces pairs of photons, which are sent in opposite directions. After the detection of the left photons, the right photon receives the information by quantum teleportation. When an interference pattern is observed, quantum teleportation (non-locality) will be denied. 
Maxwell's demon. Bell's non-locality theorem can be explained by this additional thermodynamic law. Spin is similar to a vector quantity with direction as a local hidden variable. According to Brillouin, the information of the direction can be erased because of the inevitable energy dissipation during the measurement. We proposed a determination experiment for local hidden variables, which was combined with the experiment by aspect and a double-slit experiment. When an interference pattern is observed, quantum teleportation (non-locality) will be denied. Bell's non-locality theorem can be local with this additional thermodynamic law.

\section{References}

[1] Wagner, C. (1975) Progress in Solid State Chemistry, 10, 3-16. https://doi.org/10.1016/0079-6786(75)90002-3

[2] Bouwmeester, H.J.M. and Burggraaf, A.J. (1997) Dense Ceramic Membranes for Oxygen Separator. In: Gellings, P.J. and Bouwmeester, H.J.M., Eds., The CRC Handbook of Solid State Electrochemistry, CRC Press, New York, 481-553. https://doi.org/10.1201/9781420049305.ch14

[3] Miyashita, T. (2006) Journal of Materials Science, 41, 3183-3184. https://doi.org/10.1007/s10853-006-6371-8

[4] Miyashita, T. (2011) Electrochemical and Solid-State Letters, 14, 1-4. https://doi.org/10.1149/1.3581208

[5] Miyashita, T. (2014) ECS Transactions, 59, 53-61. https://doi.org/10.1149/05901.0053ecst

[6] Miyashita, T. (2017) ECS Transactions, 75, 1-24. http://ecst.ecsdl.org/content/75/54/1.full.pdf+html

[7] Miyashita, T. (2011) Materials Sciences and Applications, 2, 180-186. https://doi.org/10.4236/msa.2011.23022

[8] Miyashita, T. (2011) Journal of Modern Physics, 2, 26-29. https://doi.org/10.4236/jmp.2011.21005

[9] Jarzynski, C. (1997) Physical Review Letters, 78, 2690-2693. https://doi.org/10.1103/PhysRevLett.78.2690

[10] Crooks, G.E. (1999) Physical Review E: Statistical Physics, Plasmas, Fluids, and Related Interdisciplinary Topics, 60, 2721-2726. https://doi.org/10.1103/PhysRevE.60.2721

[11] Sagawa, T. and Ueda, M. (2007) Jarzynski Equality with Maxwell's Demon. arXiv: cond-mat/0609085. https://arxiv.org/abs/cond-mat/0609085v3

[12] Miyashita, T. (2005) Journal of Materials Science, 40, 6027. https://doi.org/10.1007/s10853-005-4560-5

[13] Bell, J. (1964) Physics, 1, 195-200. http://cds.cern.ch/record/111654/files/vol1p195-200_001.pdf

[14] Rickert, H. (1982) Electrochemistry of Solids-An Introduction. Springer, Berlin. https://doi.org/10.1007/978-3-642-68312-1

[15] Kudo, T. (1997) Survey of Types of Solid Electrolytes. In: Gellings, P.J. and Bouwmeester, H.J.M., Eds., The CRC Handbook of Solid State Electrochemistry, CRC Press, New York, 195-221. https://doi.org/10.1201/9781420049305.ch6

[16] Dalslet, B., Blennow, P., Hendriksen, P.V., Bonanos, N., Lybye, D. and Mogensen, M. (2006) Journal of Solid State Electrochemistry, 10, 547-561. https://doi.org/10.1007/s10008-006-0135-x

[17] Boyapati, S., Wachsman, E.D. and Jiang, N. (2001) Solid State Ionics, 140, 149-160. https://doi.org/10.1016/S0167-2738(01)00698-1 
[18] Landauer, R. (1961) IBM Journal of Research and Development, 5, 183-191. https://doi.org/10.1147/rd.53.0183

[19] Gabor, D. (1964) Progress in Optics, 1, 111-153. http://www.sciencedirect.com/science/article/pii/S0079663808706097

[20] Brillouin, L. (1951) Journal of Applied Physics, 22, 334-337. https://doi.org/10.1063/1.1699951

[21] Grossing, G. (2004) Foundations of Physics Letters, 17, 343-362. https://arxiv.org/abs/quant-ph/0205047v6

[22] Grangier, A., Aspect, A. and Roger, P. (1982) Physical Review Letters, 49, 91-94. https://doi.org/10.1103/PhysRevLett.49.91

Submit or recommend next manuscript to SCIRP and we will provide best service for you:

Accepting pre-submission inquiries through Email, Facebook, LinkedIn, Twitter, etc. A wide selection of journals (inclusive of 9 subjects, more than 200 journals)

Providing 24-hour high-quality service

User-friendly online submission system

Fair and swift peer-review system

Efficient typesetting and proofreading procedure

Display of the result of downloads and visits, as well as the number of cited articles Maximum dissemination of your research work

Submit your manuscript at: http://papersubmission.scirp.org/

Or contact jmp@scirp.org 